CORRECTION

https://doi.org/10.1038/s41586-019-0959-z

\title{
Publisher Correction: Actively personalized vaccination trial for newly diagnosed glioblastoma
}

Norbert Hilf, Sabrina Kuttruff-Coqui, Katrin Frenzel, Valesca Bukur, Stefan Stevanović, Cecile Gouttefangeas, Michael Platten, Ghazaleh Tabatabai, Valerie Dutoit, Sjoerd H. van der Burg, Per thor Straten, Francisco Martinez-Ricarte, Berta Ponsati, Hideho Okada, Ulrik Lassen, Arie Admon, Christian H. Ottensmeier, Alexander Ulges, Sebastian Kreiter, Andreas von Deimling, Marco Skardelly, Denis Migliorini, Judith R. Kroep, Manja Idorn, Jordi Rodon, Jordi Piro, Hans S. Poulsen, Bracha Shraibman, Katy McCann, Regina Mendrzyk, Martin Lower, Monika Stieglbauer, Cedrik M. Britten, David Capper, Marij J. P. Welters, Juan Sahuquillo, Katharina Kiesel, Evelyna Derhovanessian, Elisa Rusch, Lukas Bunse, Colette Song, Sandra Heesch, Claudia Wagner, Alexandra Kemmer-Bruck, Jorg Ludwig, John C. Castle, Oliver Schoor, Arbel D. Tadmor, Edward Green,

Jens Fritsche, Miriam Meyer, Nina Pawlowski, Sonja Dorner, Franziska Hoffgaard, Bernhard Rossler, Dominik Maurer, Toni Weinschenk, Carsten Reinhardt, Christoph Huber, Hans-Georg Rammensee, Harpreet Singh-Jasuja, Ugur Sahin, Pierre-Yves Dietrich \& Wolfgang Wick

Correction to: Nature https://doi.org/10.1038/s41586-018-0810-y, published online 19 December 2018.

In this Letter, owing to an error in the production process, the additional author support information was omitted from the Supplementary Information. The Supplementary Information of the original Letter has been corrected online. 